\title{
Further application of the deconvolution method of post-depositional DRM to the precise record of the Matuyama-Brunhes reversal in the sediments from the Boso Peninsula, Japan
}

\author{
Hideo Tsunakawa ${ }^{1}$, Makoto Okada ${ }^{2}$, and Nobuaki Niitsuma ${ }^{3}$ \\ ${ }^{1}$ Department of Earth and Planetary Sciences, Tokyo Institute of Technology, Tokyo 152-8551, Japan \\ ${ }^{2}$ Department of Earth Sciences, Ibaraki University, Mito, Ibaraki 310-8512, Japan \\ ${ }^{3}$ Department of Geosciences, Shizuoka University, Shizuoka 422-8529, Japan
}

(Received June 22, 1998; Revised November 26, 1998; Accepted December 25, 1998)

\begin{abstract}
The Matuyama-Brunhes geomagnetic reversal was continuously recorded in the massive siltstones of the Boso marine sediments in Japan with the time resolution better than 9 years. However the Boso sediments probably have the post-depositional detrital remanent magnetization (pDRM) depressing short-period variations due to the convolution of the geomagnetic field. The previous application of the deconvolution method of pDRM to the 900 year records gave the half fixing depth of $21 \mathrm{~cm}$. Further application of this fixing depth to the 4100 year record clearly shows nearly 100 year variations in both of the inclination and declination.
\end{abstract}

\section{Introduction}

The paleomagnetic measurements of the BrunhesMatuyama transition field at the Yanagawa site $\left(35^{\circ} 15^{\prime} \mathrm{N}\right.$, $\left.140^{\circ} 15^{\prime} \mathrm{E}\right)$ have been performed for the continuous section of about $15 \mathrm{~m}$ thickness (Niitsuma, 1971; Okada and Niitsuma, 1989). Adapting the accumulation rate of $3.7 \mathrm{~mm} /$ year, which was estimated mainly from the oxygen isotope study (Okada and Niitsuma, 1989), this span is considered to cover about 4100 years. All the span belongs to the transition of the Matuyama-Brunhes reversal defined from relatively weak field intensities (Fig. 1). Two or three specimens of $35 \mathrm{~mm}$ in diametre were drilled from the outcrop and cut into $32 \mathrm{~mm}$ in length, so that the measurement of each specimen is averaged for 8.6 year interval. The viscous component is mostly removed in alternating field demagnetization by $20 \mathrm{mT}$. The reconnaissance survey (Niitsuma, 1971) of the Boso paleomagnetism reported the remanence directions after $9 \mathrm{mT}$ demagnetization, but its magnetic cleaning seems to be insufficient. We analyzed the directions at $2 \mathrm{~cm}$ (5.4 year) intervals obtained from the Bayesian smoothing (Tsunakawa, 1992) of 1295 original data after $25 \mathrm{mT}$ magnetic cleaning (Fig. 1).

The previous spectral analysis (Tsunakawa et al., 1996) suggested the periodic changes of the order of 100 years in the field directions during the Matuyama-Brunhes reversal. However it is widely accepted that the pDRM effect should be taken into account for the sedimentary magnetism, especially for the study on the rapid change in the geomagnetic field. Because the pDRM mechanism has not yet completely been clarified, most of the paleomagnetic studies have not removed the effect of pDRM from the reversal records except for a few reports (Okada and Niitsuma, 1989; Kent and

Copy right (C) The Society of Geomagnetism and Earth, Planetary and Space Sciences (SGEPSS); The Seismological Society of Japan; The Volcanological Society of Japan; The Geodetic Society of Japan; The Japanese Society for Planetary Sciences.
Schneider, 1995; Tsunakawa et al., 1996). As the pDRM origin is inferred for the Boso sedimentary magnetism from the evidence of intensive bioturbation (Okada and Niitsuma, 1989; Tsunakawa et al., 1995), we have to restore more accurate variations from the measured remanence directions.

Tsunakawa et al. (1996) applied the deconvolution method (Tsunakawa, 1995) to the two directional datasets of about 900 years from the different sites, Yanagawa and Heizogawa. As a result, the half fixing depth of an exponential fixing function in pDRM acquisition (Hyodo, 1984) was estimated to be $21 \pm 1(1 \sigma) \mathrm{cm}$ for the Boso sediments, which seems reasonable in comparison with those for other marine sediments (Tsunakawa et al., 1996). The 4100 year dataset of Yanagawa, analyzed in the present study, includes the 900 year data section and the half fixing depth is assumed to be constant $(21 \mathrm{~cm})$ throughout the span. The paleomagnetic directions are attempted to be deconvolved with the technique proposed by Tsunakawa et al. (1996).

The deconvolution technique with an exponential fixing function is explained below for a single directional dataset from sediments, when a fixing depth $(Z)$ is known.

The fixing factor $(A)$ is defined to be,

$$
A=(z / Z) \ln 2,
$$

where $z$ is a sedimentation rate. Directions of sedimentary magnetization and the original geomagnetic fields are expressed as $\boldsymbol{m}_{j}$ and $\boldsymbol{f}_{j}(j=1, \ldots, n-1)$ at time intervals of $\Delta t$, respectively.

$$
\begin{gathered}
\boldsymbol{f}_{j}=c^{-} \boldsymbol{m}_{j}+c^{+} \boldsymbol{m}_{j+1}, \\
c^{ \pm}=1 / 2 \pm 1 /(A \Delta t),
\end{gathered}
$$

where we suppose that intensities gradually change rather than directions. This calculation is a very simple recursive type and basically has no assumptions of the frequency dependence in the original field, while the FFT method 
Inc. [deg]

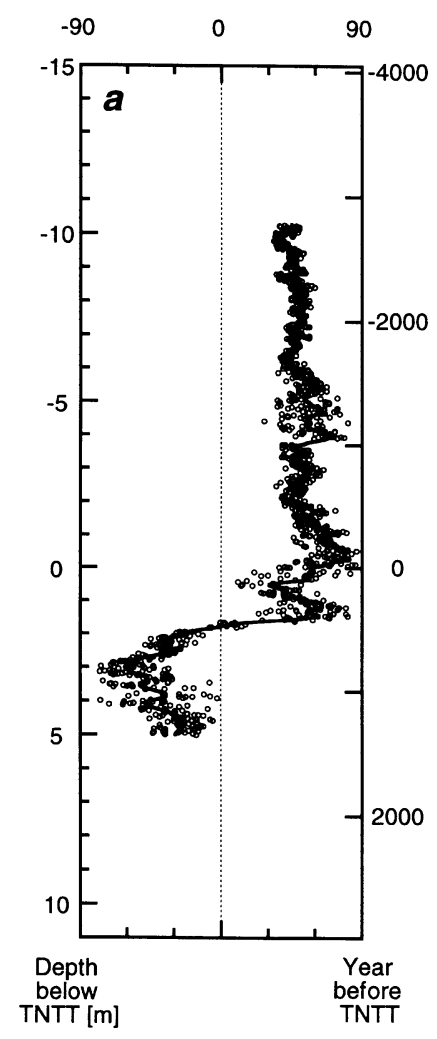

Dec. [deg]

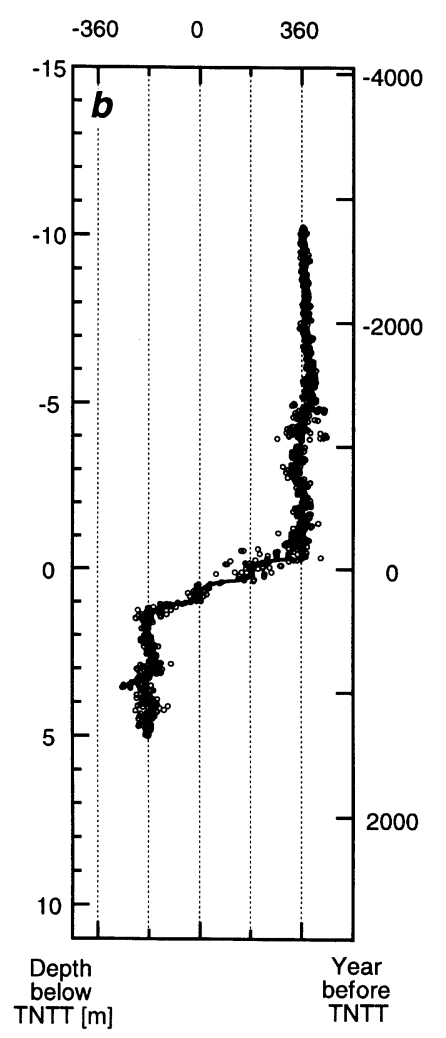

NRM/ARM

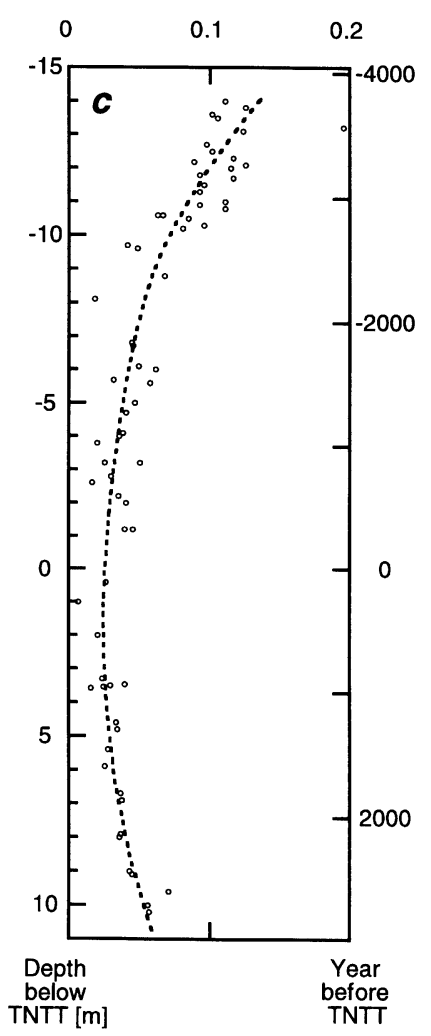

Fig. 1. The original paleomagnetic data from the Boso marine sediments recording the Bruhnes-Matuyama reversal (Niitsuma, 1971; Okada and Niitsuma, 1989). The inclination in the left column and the declination in the middle were smoothed by the solid curves in the Bayesian procedure (Tsunakawa, 1992; Tsunakawa et al., 1995). Available data of the NRM/ARM ratio are also shown in the right diagram as relative intensity changes, which are calculated from the demagnetization results by $40 \mathrm{mT}$ (Okada and Niitsuma, 1989) and smoothed with a broken line (Tsunakawa, 1992). Precise measurements of magnetization directions have been performed for about 4100 years. The relative age is defined against the deposition of the key ash bed, TNTT (Niitsuma, 1971).

(Hyodo, 1984) assumes that the geomagnetic field really has frequency components approximated by the finite Fourie transform. The details are discussed in Tsunakawa et al. (1995).

The deconvolved directions were analyzed with the spectral analysis to examine nearly 100 year variations. Although the dataset has been sophisticatedly processed with smoothing and deconvolution, we believe that the characteristic features can be detected.

\section{Results and Discussion}

The deconvolution results are shown in Fig. 2. The shortperiod variations of about 100 years are clearly recognized in both of the inclination and declination after the deconvolution. As shown by Tsunakawa (1995), the frequency response of the present deconvolution is very smooth and then any specific frequency component is not newly generated. Therefore these variations are believed not to be an artifact. From the behavior of field directions in Fig. 2, the span is divided into four stages; (1) reverse direction, (2) main reversal, (3) rebound zone, and (4) normal direction. The duration of intermediate directions is about 200 and 500 years in the main reversal and in the rebounds, respectively. The equal-area projections of VGPs are also shown for the four stages in Fig. 3, respectively. The full reversal and re- bound give the weak longitudinal confinement of VGP paths (Clement, 1991; Laj et al., 1991) around $120^{\circ}$ but longitudinal drift seems to be dominant in the polar equal area projection (Fig. 3). The spatial distribution of transitional VGPs seems to have a relatively dense patch near Australia as indicated from volcanic rocks (Hoffman, 1991) and from drift deposits (Channell and Lehman, 1997).

The first derivatives of the inclination $(\Delta I)$ and the modified declination $(\Delta D \cos I)$, removing long-term trends from the deconvolved directions, are used for the spectral analysis (Fig. 2). The maximum rate of angular changes in the deconvolved directions is $3 \mathrm{deg} /$ year at the age of 80 years before the deposition of the key ash bed of TNTT (Niitsuma, 1971). The maximum entropy method (MEM) was applied using a window of 100 data points (540 years) with a shift of 50 points ( 270 years). The length of the prediction error filter $(M)$ was determined by minimizing an information criterion, AIC (Akaike, 1969, 1973). The AIC shows the minimum for $M \leq 10$ and its length appears to be short when compared with the number of data points. This is probably due to removal of noises by the smoothing and then the spectral analysis may have a relatively lower resolution. The results are shown in Fig. 4 together with the VGP latitude diagram. Most of the power spectra have a single peak around 100 years for both of the inclination and declination while sev- 

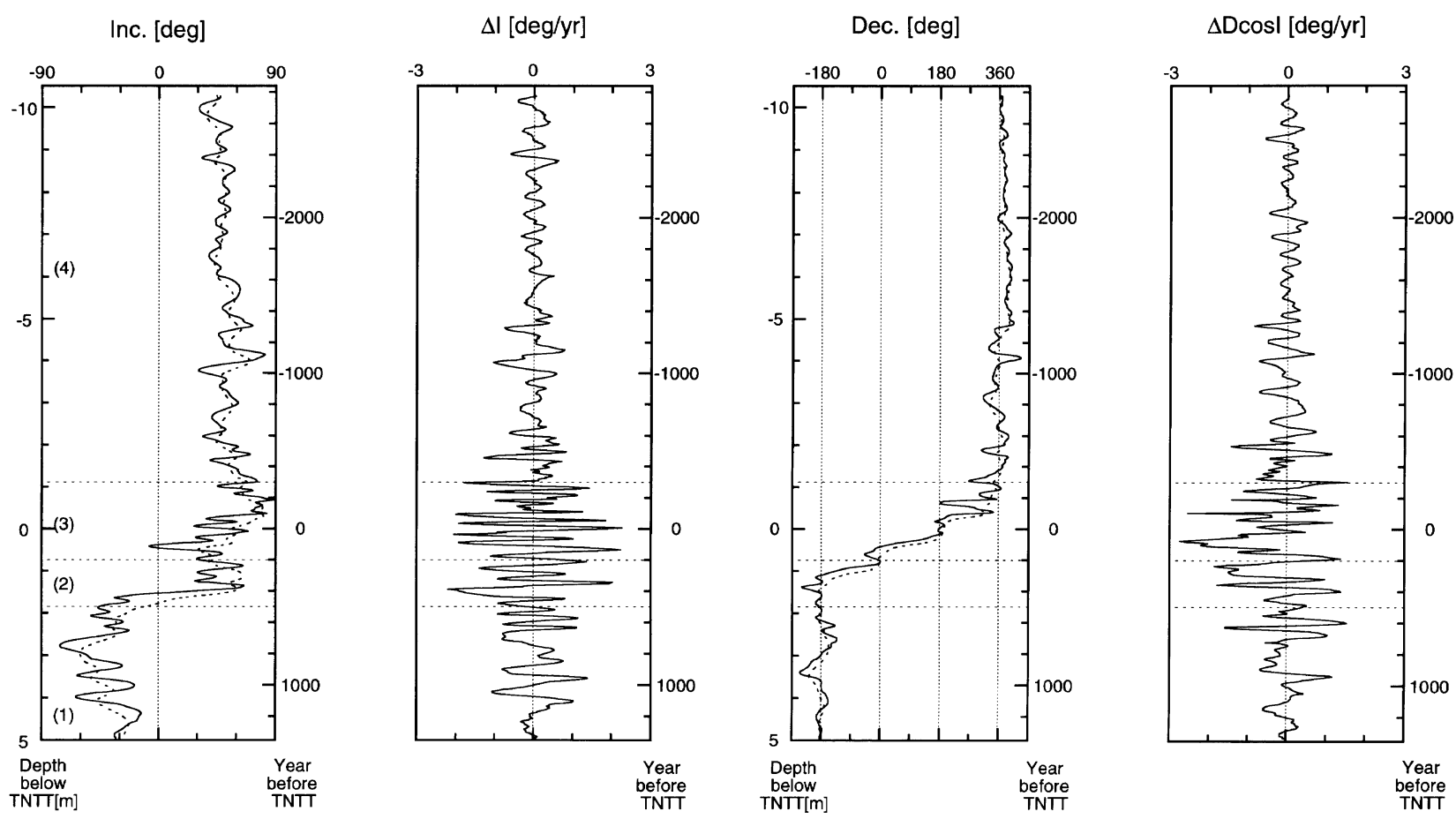

Fig. 2. Directional changes before (dotted lines) and after (solid lines) the deconvolution from the Boso paleomagnetism. The first derivatives of the inclination $(\Delta I)$ and the modified declination $(\Delta D \cos I)$ after the deconvolution are also shown. The span is divided into four stages; (1) reverse direction, (2) main reversal, (3) rebound zone, and (4) normal direction.
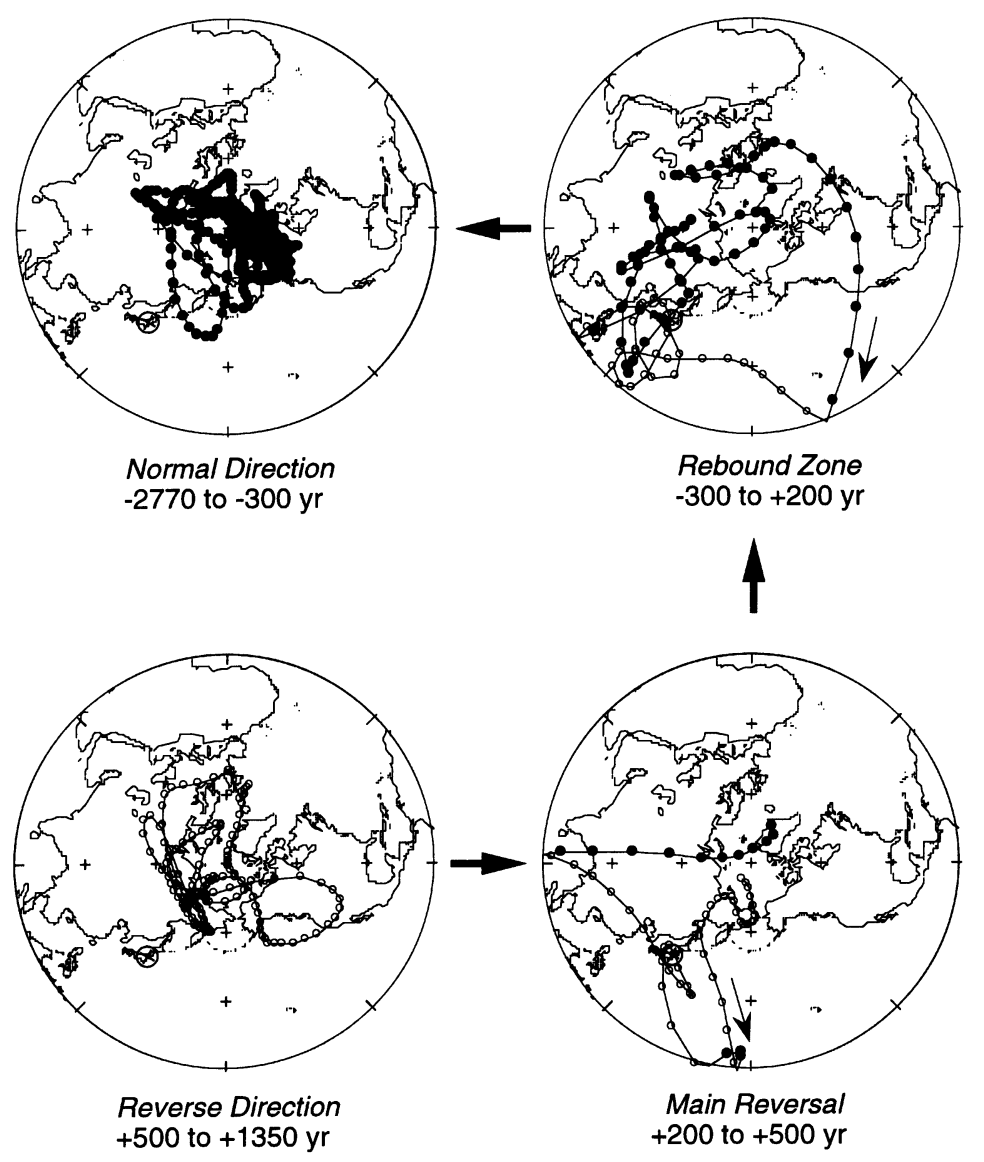

Fig. 3. Equal-area projection from the north pole of the VGP paths calculated from the deconvolved directions (see Fig. 2). The circle represents a VGP at 5.4 year intervals. Open circles: in the southern hemisphere, closed circles: in the northern hemisphere, cross: the sampling site. 

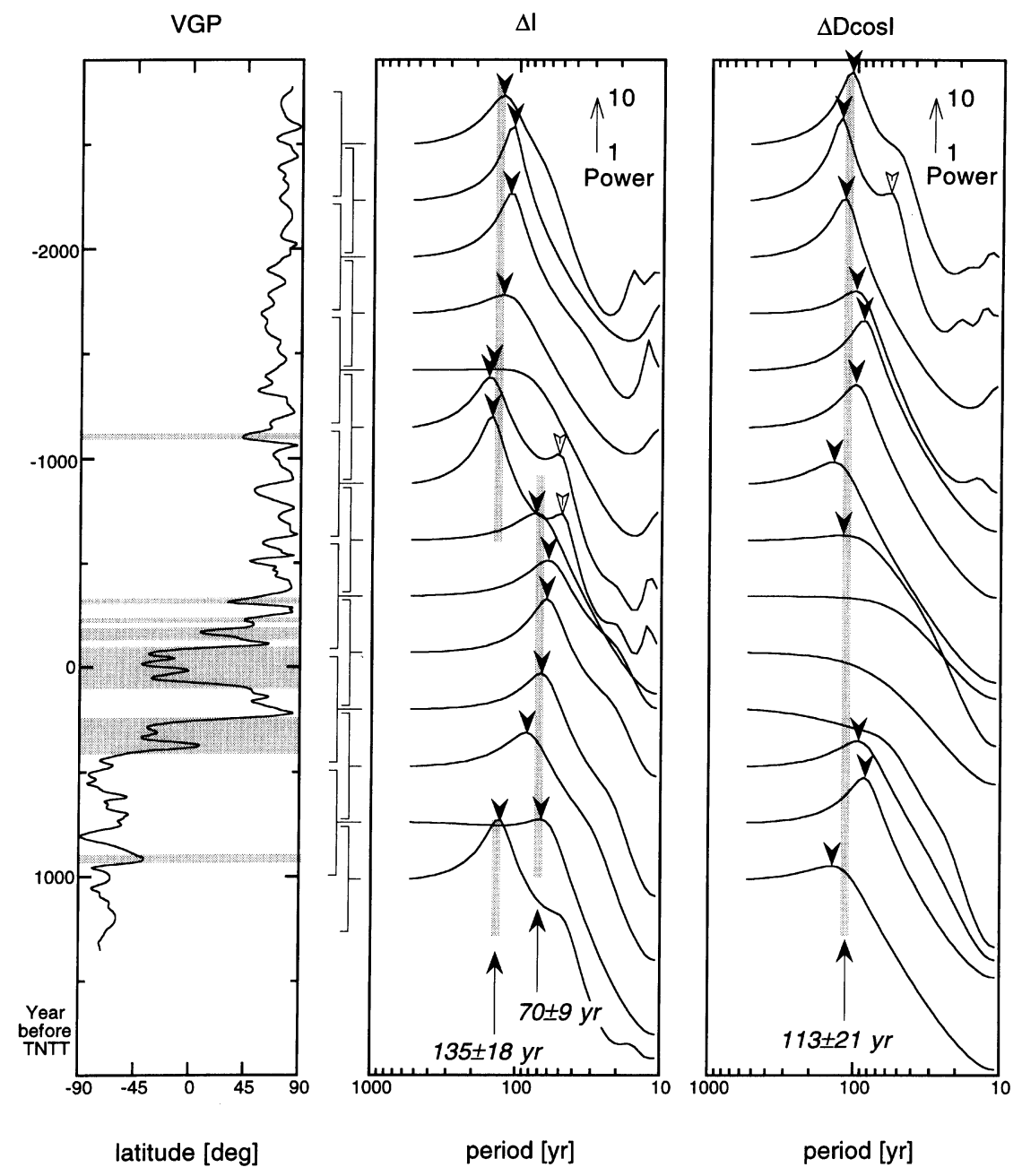

Fig. 4. Power spectra of the directional variations in the first derivatives (see Fig. 1). The curves represent the logarithmic power spectrum for 540 year successive intervals with a 270 year shift. The applied data windows are shown between the left and the middle diagrams. Solid arrow: the first peak of the power, open arrow: the second peak. The VGP latitudes are also shown in the left column, where the hatched zone indicates the intermediate position between $45^{\circ} \mathrm{N}$ and $45^{\circ} \mathrm{S}$.

eral spectra show a second peak or a flat pattern. Hence, it is concluded that the short-period variations were of the order of 100 years during about 4100 years of the transitional state.

The three-dimensional (3D) simulation has recently demonstrated a self-sustaining dynamo associated with a full reversal (Glatzmaier and Roberts, 1995a,b). The 3D geodynamo also indicated that a magnetic field in the fluid outer core is attempting to change its axial dipole polarity on a short time scale $(\sim 100$ years) though the inner-core field persists its polarity, resulting in a stable state (Glatzmaier and Roberts, 1995b). This variation in the outer-core field may be relevant to the nearly 100 year periodicity observed in this paleomagnetic study.

The first peak of the inclination spectrum has shorter periods in the stages of the main reversal and the subsequent rebound zone than in other stages (Fig. 4). The average is calculated to be $70 \pm 9(1 \sigma)$ years for the shorter periods and $135 \pm 18(1 \sigma)$ years for the longer. There is a possibility that this difference is not significant because it comes from the smoothed curves. However the systematic change in the pe- riodicity suggests that two states of the core dynamics might possibly have generated two different variations during the Matuyama-Brunhes reversal. On the other hand, the declination spectral peaks seem to have no systematic changes and the average for all is $113 \pm 21$ years. One may speculate that some zonal component(s) controlled the inclination with 70 and 135 year periodic changes and some nonzonal component(s) did the declination through a 113 year oscillation. However, because we have a dataset from a single site, it is difficult to identify which component controls these periodic variations.

Nearly 100 year period observed in this study is of the same order as that of the recent secular variations ( $\sim 60$ years $)$ in the Gauss coefficients, especially for the terms of degree $\leq 4$ (Yokoyama and Yukutake, 1991; Langel et al., 1986) though they have not been established yet. Although the amplitude seems different, the paleomagnetic reversal record, dynamo simulation and the recent geomagnetic field would be relevant to each other regarding the mechanism of the short-period variations. 


\section{Conclusions}

The Matuyama-Brunhes geomagnetic reversal with the time resolution better than 9 years was analyzed by the new deconvolution technique of pDRM. Spatial distributions of VGPs suggests the weak confinement around $120^{\circ}$ and denser patch near Australia. Spectral analysis of the inclination and declination clearly shows about 100 year periodicity, which is similar to that of the outer-core field fluctuations inferred from the recent three-dimensional geodynamo simulation and that of the recent geomagnetic secular variation.

Acknowledgments. We thank Dr. H. Shimizu, University of Tokyo, and an anonymous reviewer for their helpful comments.

\section{References}

Akaike, H., Power spectrum estimation through autoregressive model fitting, Ann. Inst. Statist. Math., 21, 407-419, 1969.

Akaike, H., Information theory and an extension of the maximum likelihood principles, in 2nd Int. Symp. on Information Theory, edited by B. N. Petrov and F. Csaki, pp. 267-281, 1973.

Channell, J. E. T. and B. Lehman, The last two geomagnetic polarity reversals recorded in high-deposition-rate sediment drifts, Nature, $\mathbf{3 8 9}$, 712-715, 1997.

Clement, B. M., Geographical distribution of transitional VGPs: evidence for non-zonal equatorial symmetry during the Matuyama-Brunhes geomagnetic reversal, Earth Planet. Sci. Lett., 104, 48-58, 1991.

Glatzmaier, G. A. and P. H. Roberts, A three-dimensional self consistent computer simulation of a geomagnetic field reversal, Nature, 377, 203209, 1995a.

Glatzmaier, G. A. and P. H. Roberts, A three-dimensional convective dynamo solution with rotating and finitely conducting inner core and mantle, Phys. Earth Planet. Inter, 91, 63-75, 1995b.

Hoffman, K. A., Long-lived transitional states of the geomagnetic field and the two dynamo families, Nature, 354, 273-277, 1991.
Hyodo, M., Possibility of reconstruction of the past geomagnetic field from homogeneous sediments, J. Geomag. Geoelectr., 36, 45-62, 1984.

Kent, D. V. and D. A. Schneider, Correlation of paleointensity variation records in the Brunhes/Matuyama polarity transition interval, Earth Planet. Sci. Lett., 129, 135-144, 1995.

Laj, C., R. Mazaud, R. Weeks, M. Fuller, and E. Herrero-Bervera, Geomagnetic reversal paths, Nature, 351, 447, 1991.

Langel, R. A., D. J. Kerridge, D. R. Barraclough, and S. R. Malin, Geomagnetic temporal change: 1903-1982, a spline representation, J. Geomag. Geoelectr., 38, 573-597, 1986.

Niitsuma, N., Detailed study of the sediments recording the MatuyamaBrunhes geomagnetic reversal, Tohoku Univ. Sci. Rep., 2nd Ser. (Geol.), 43, 1-39, 1971.

Okada, M. and N. Niitsuma, Detailed paleomagnetic records during the Matuyama-Brunhes geomagnetic reversal and a direct determination of depth lag for magnetization in marine sediments, Phys. Earth Planet. Inter, 56, 133-150, 1989.

Tsunakawa, H., Bayesian approach to smoothing palaeomagnetic data using ABIC, Geophys. J. Int., 108, 801-811, 1992.

Tsunakawa, H., Deconvolution method for directions of the post-depositional detrital remanent magnetization using an exponential fixing function, J. Geomag. Geoelectr., 47, 551-576, 1995.

Tsunakawa, H., M. Okada, and N. Niitsuma, About 100 year directional variations in the Matuyama-Brunhes transition field inferred from the sedimentary records in the Boso Peninsula, Japan, J. Geomag. Geoelectr., 47, 337-345, 1995.

Tsunakawa, H., M. Okada, and N. Niitsuma, Deconvolution of paleomagnetic directions from marine sediments in the Boso Peninsula, Japan, and its implication for the Matuyama-Brunhes transitional field, J. Geomag. Geoelectr., 48, 1541-1552, 1996.

Yokoyama, Y. and H. Yukutake, Sixty year variation in a time series of the geomagnetic Gauss coefficients between 1910 and 1983, J. Geomag. Geoelectr., 43, 563-584, 1991.

H. Tsunakawa (e-mail: htsuna@geo.titech.ac.jp), M. Okada, and N. Niitsuma 